\title{
Cochrane
}

Library

Cochrane Database of Systematic Reviews

\section{Transient elastography with controlled attenuation parameter (CAP) for diagnosis of moderate or severe steatosis in people with suspected non-alcoholic fatty liver disease (Protocol)}

Turankova T, Blyuss O, Brazhnikov A, Svistunov A, Gurusamy KS, Pavlov CS

Turankova T, Blyuss O, Brazhnikov A, Svistunov A, Gurusamy KS, Pavlov CS.

Transient elastography with controlled attenuation parameter (CAP) for diagnosis of moderate or severe steatosis in people with suspected non-alcoholic fatty liver disease (Protocol).

Cochrane Database of Systematic Reviews 2020, Issue 7. Art. No.: CD013670.

DOI: 10.1002/14651858.CD013670.

www.cochranelibrary.com

Transient elastography with controlled attenuation parameter (CAP) for diagnosis of moderate or severe steatosis in people with suspected non-alcoholic fatty liver disease (Protocol)

Copyright $\odot 2020$ The Cochrane Collaboration. Published by John Wiley \& Sons, Ltd. 
HEADER 1

ABSTRACT

BACKGROUND

Figure 1.

OBJECTIVES

METHODS

ACKNOWLEDGEMENTS

REFERENCES

ADDITIONAL TABLES

APPENDICES

WHAT'S NEW

HISTORY

CONTRIBUTIONS OF AUTHORS

DECLARATIONS OF INTEREST

SOURCES OF SUPPORT

\section{TABLE OF CONTENTS}




\section{Transient elastography with controlled attenuation parameter (CAP) for diagnosis of moderate or severe steatosis in people with suspected non- alcoholic fatty liver disease}

Taisiia Turankova1 ${ }^{1}$, Oleg Blyuss²,3,4, Alexey Brazhnikov5, Andrey Svistunov6 ${ }^{6}$ Kurinchi Selvan Gurusamy1,7, Chavdar S Pavlov 1,8

1Department of Therapy, I.M. Sechenov First Moscow State Medical University, Moscow, Russian Federation. 2 School of Physics, Astronomy and Mathematics, University of Hertfordshire, Hatfield, UK. ${ }^{3}$ Department of Paediatrics and Paediatric Infectious Diseases, I.M. Sechenov First Moscow State Medical University, Moscow, Russian Federation. ${ }^{4}$ Department of Applied Mathematics, Lobachevsky State University of Nizhny Novgorod, Nizhny Novgorod, Russian Federation. 5Department of Epidemiology and Evidence-Based Medicine, I.M. Sechenov First Moscow State Medical University, Moscow, Russian Federation. 6I.M. Sechenov First Moscow State Medical University, Moscow, Russian Federation. ${ }^{7}$ Division of Surgery and Interventional Science, University College London, London, UK. ${ }^{8}$ Cochrane Hepato-Biliary Group, Copenhagen Trial Unit, Centre for Clinical Intervention Research, Department 7812, Rigshospitalet, Copenhagen University Hospital, Copenhagen, Denmark

Contact address: Chavdar S Pavlov, chpavlov@mail.ru.

Editorial group: Cochrane Hepato-Biliary Group.

Publication status and date: Edited (no change to conclusions), published in Issue 8, 2020.

Citation: Turankova T, Blyuss O, Brazhnikov A, Svistunov A, Gurusamy KS, Pavlov CS. Transient elastography with controlled attenuation parameter (CAP) for diagnosis of moderate or severe steatosis in people with suspected non-alcoholic fatty liver disease (Protocol). Cochrane Database of Systematic Reviews 2020, Issue 7. Art. No.: CD013670. DOI: 10.1002/14651858.CD013670.

Copyright @ 2020 The Cochrane Collaboration. Published by John Wiley \& Sons, Ltd.

\section{A B S T R A C T}

\section{Objectives}

This is a protocol for a Cochrane Review (diagnostic). The objectives are as follows:

To determine the diagnostic accuracy of transient elastography with CAP for diagnosis of moderate and severe hepatic steatosis in people with suspected NAFLD when compared with liver biopsy as reference standard. To achieve this, we will compare none/mild hepatic steatosis ( $\mathrm{S} 0$ to $\mathrm{S} 1$ ) versus moderate/severe hepatic steatosis (S2 to S3); and none/mild/moderate hepatic steatosis (S0 to S2) versus severe hepatic steatosis (S3).

\section{Secondary objectives}

We aim to also identify the pooled sensitivity and specificity for the most common cut-off values of CAP for diagnosis of none/mild hepatic steatosis (S0 to S1) and moderate/severe hepatic steatosis (S2 to S3); or none/mild/moderate hepatic steatosis (S0 to S2) and severe hepatic steatosis (S3) in people with suspected NAFLD, and to explore potential sources of heterogeneity influencing the diagnostic test accuracy of CAP (see: Investigations of heterogeneity). 


\section{B A C K G R O U N D}

Hepatic steatosis (i.e. abnormal retention of fat) is the main clinical manifestation of non-alcoholic fatty liver disease (NAFLD) (Tiniakos 2018). Whenever there is a suspicion of NAFLD, hepatic steatosis should be documented and measured. Steatosis is defined as intrahepatic fat of at least $5 \%$ of liver weight (EASL Guidelines 2016). Liver biopsy has been the primary method for diagnosing NAFLD, and it is the most accurate method for assessing steatosis (EASL Guidelines 2016). The assessment of steatosis is based on measuring the extent of the surface area of a histological section involved, or uninvolved, in the parenchyma, providing the percentage of the affected area. Histologically, hepatic steatosis is graded from 0 to 3: minimal steatosis (grade S0), defined as presence of less than $5 \%$ or $10 \%$; mild steatosis, between $5 \%$ and $33 \%$ (grade S1); moderate steatosis, between 33\% and 66\% (grade S2); and severe steatosis, more than $66.0 \%$ (grade S3) (Table 1 ).

The pathological spectrum of NAFLD ranges from simple steatosis to non-alcoholic steatohepatitis (NASH), fibrosis, cirrhosis, and hepatocellular carcinoma (HCC) (Angulo 2002). Diagnosis of NAFLD is also based on lack of secondary causes of hepatic fat accumulation: ruling out excessive alcohol consumption, viral hepatitis, other chronic liver diseases such as Wilson's disease, haemochromatosis, autoimmune hepatitis, cholestatic liver disease, starvation, lipodystrophy, coeliac disease, or Cushing's disease as well as medications (corticosteroids, methotrexate, diltiazem, oxaliplatin, amiodarone, isoniazid, highly active antiretroviral therapy, etc.) (Perumpail 2017). Clinical guidelines suggest moderate consumption of alcohol as a component of NAFLD diagnosis. In Chalasani 2012, EASL Guidelines 2016, NICE 2016, and AISF 2017 guidelines, it is less than $30 \mathrm{~g} /$ day for men and less than $20 \mathrm{~g} /$ day for women; in the American Association for the Study of Liver Diseases (AASLD) guidelines it is fewer than 21 standard drinks/week in men and fewer than 14 in women (Chalasani 2018); and in the Asia-Pacific Guidelines, it is less than 14 standard alcoholic drinks/week in men and 14 in women (Chitturi 2018; Wong 2018).

Non-alcoholic hepatic steatosis is associated with obesity, type 2 diabetes mellitus (T2DM), and dyslipidaemia. Most often, steatosis is detected for the first time when an NAFLD is established. There seems to be no isolated data on the frequency and prognosis of steatosis. One meta-analysis on data of people with NAFLD from 1989 to 2015 found a global prevalence of 25.2\% (Younossi 2016). Specifically, the prevalence of NAFLD was highest in the Middle East (31\%) and South America (32\%), and lowest in Africa (13.5\%) (Younossi 2016).

Among histological features, only liver fibrosis is associated with long-term outcomes of people with NAFLD (Angulo 2015; Ekstedt 2015). On baseline biopsy, people with NAFLD, but without fibrosis, exhibited progression of fibrosis of one-stage over 14 years on average, whereas those with NASH progressed to fibrosis with one stage over seven years (Singh 2015). McPherson 2015 reported that progression of NAFLD occurred irrespective of whether the first biopsy showed hepatic steatosis or NASH; about $20 \%$ of people showed rapid fibrosis, whereas the remaining $80 \%$ had minimal progression. Another study reported that, compared to the general population, NAFLD was associated with an increased overall mortality (standardised mortality ratio of 1.34 to 2.6) (Kwak 2015). Adams 2005 and Ekstedt 2006 reported an absolute risk of liver-related mortality being $1.7 \%$ (median follow-up of 8 to 14 years), while Soderberg 2010 reported liver-related mortality of $9.6 \%$ (median follow-up of 21 years). Based on these studies, Angulo 2013 concluded that "the overall and liver-related mortality in patients with the whole spectrum of NAFLD is greater than in the general population of same age and gender, and that complications of end-stage liver disease contribute significantly to this higher mortality."

There are no drugs currently approved to prevent or treat hepatic steatosis. A number of pharmacological interventions have been tried in NAFLD, but with overall limited benefit. Lifestyle modification consisting of diet, exercise, and weight loss have been advocated to treat people with NAFLD. Foregut bariatric surgery can also be considered an option to specifically treat obese people with NAFLD or NASH (Chalasani 2018). Traditionally, liver biopsy has been the primary method for diagnosing and assessing the severity of NAFLD (EASL Guidelines 2016). However, when a disease and its progression should be monitored, liver biopsy is not of a practical use, also because it is an invasive procedure and carries a small, but definite, risk of complications. Transient elastography with controlled attenuation parameter (CAP) is an appropriate alternative to liver biopsy in diagnosing steatosis, identification of risk groups, and in evaluation of response to lifestyle modifications (Lombardi 2017; Gurusamy 2018).

\section{Target condition being diagnosed}

The target conditions are moderate or severe hepatic steatosis (i.e. S2 and S3) in people with suspected NAFLD. Moderate steatosis (S2) is classified by pathology when it is between $33 \%$ and $66 \%$, and as severe (S3) when it is more than $66 \%$.

\section{Index test(s)}

\section{Transient elastography with controlled attenuated parameter}

Transient elastography with CAP is a suitable diagnostic tool to detect and quantify steatosis along with measuring liver fibrosis (de Ledinghen 2014).

Transient elastography is a mechanical test designed to measure liver stiffness (i.e. the health of the liver) in kilopascals. Steatosis quantification by CAP analyses a measure of the ultrasound attenuation, which corresponds to the decrease in amplitude of ultrasound waves as they propagate through the liver (Echosens 2011). The technology is based on the evaluation of ultrasound signals acquired using a FibroScan device (an ultrasoundbased vibration-controlled transient elastography (VCTE) system) (Sandrin 2002). A probe is put on the skin surface overlying the liver. After pressing the button on the probe, a pulse wave is transmitted across the liver parenchyma. After a short interval, a second ultrasound wave is transmitted, and the difference between the velocity of the two waves in the liver parenchyma is calculated. Intrahepatic cholestasis, ascites, and congestive heart failure are factors that can distort the results. VCTE system is contraindicated for pregnant women and people with active implantable medical devices (Echosens 2011). CAP is measured with a probe $M$, designed for the general adult population, at $3.5 \mathrm{MHz}$ at a depth between $25 \mathrm{~mm}$ and $65 \mathrm{~mm}$, or with a probe XL, designed for overweight people, at a depth between $35 \mathrm{~mm}$ and $75 \mathrm{~mm}$. The $\mathrm{XL}$ probe was developed because of problems of obtaining valid measurements with the M-probe in people with an increased skin-liver length, which was a frequent finding in obese people (de Ledinghen 2010). 
CAP is calculated simultaneously with liver stiffness only if the liver stiffness measurements are valid (i.e. range between 100 decibel per metre $(\mathrm{dB} / \mathrm{m})$ and $400 \mathrm{~dB} / \mathrm{m})$. If they are not valid (i.e. within the provided range), then CAP is not measured (Echosens 2011). Measurement confidence is defined as 1.10 valid measurements or more; 2 . interquartile range/median (IQR/med) less than $30 \%$ in elastography; and 3. success rate greater than 60\% (Castera 2010).

The advantages of transient elastography with CAP are that it is a non-invasive test, easy to perform, provides immediate results, and has low cost in comparison with other measurement modalities, such as computed tomography or magnetic resonance-based methods (de Ledinghen 2014).

One study by Sasso 2010, including 115 participants with chronic liver disease from various causes, was the first to validate CAP as a diagnostic tool for detection and quantification of steatosis, using liver biopsy as the reference standard. Subsequently, strong correlation of CAP with steatosis in the liver compared with liver biopsy has been shown for many chronic liver diseases (Table 2). Diagnostic accuracy of transient elastography with CAP may be affected by variations in cut-off values of different steatosis grades and different covariates (de Ledinghen 2014). Factors associated with elevated CAP are body mass index (BMI), metabolic syndrome, alcohol, and liver stiffness (Karlas 2017). Also, accurate diagnosis of the stage of steatosis makes it possible to evaluate the risk of developing cardiovascular disease, prognosis, and effectiveness of treatment, which in particular can increase the patient's compliance to further treatment.

\section{Clinical pathway}

People with insulin resistance or metabolic risk factors, or both (i.e. obesity or metabolic syndrome, age more than 50 years) as well as with persistently abnormal liver enzymes should be suspected of having NAFLD. International guidelines partly differ in the diagnosis of hepatic steatosis in terms of acceptable economic burden (Table 3). The optimal strategy for detecting and grading steatosis, as well as stratifying people with suspected NAFLD and the way to followup the disease progression, has not yet been established as there are no pathognomonic symptoms or signs that can be used for the diagnosis (Leoni 2018).

The non-invasive assessment of steatosis in people with suspected NAFLD includes clinical, laboratory, and imaging tests to diagnose NAFLD. A slight increase in the level of aspartate aminotransferase (AST), alanine aminotransferase (ALT), or gamma-glutamyl transpeptidase (GGTP) can be observed in laboratory data, but normal levels of liver enzymes do not exclude absence of NAFLD. International guidelines vary in their recommendations on how to confirm the presence of steatosis and general liver biochemistry in people with suspected NAFLD. As an initial diagnostic workup, some guidelines recommend non-invasive imaging examinations, the sequence of which in practice may depend on the availability and cost (EASL Guidelines
2016; NICE 2016; AISF 2017; Chalasani 2018; Chitturi 2018; Wong 2018). The EASL Guidelines 2016, Asia-Pacific (Chitturi 2018; Wong 2018), and Italian (AISF 2017) clinical guidelines suggest the Fatty Liver Index (FLI) and the NAFLD liver fat score for assessing the presence of steatosis. The AASLD guidelines underline the lack of evidence for the usefulness of quantifying liver steatosis in routine clinical settings and highlight that only the grade of inflammation or fibrosis can determine the prognosis of people with NAFLD (Chalasani 2018). All guidelines previously mentioned agree on the need of non-invasive laboratory and imaging assessment of advanced fibrosis as the leading prognostic factor among people with NAFLD due to a strong correlation with survival rate and liverrelated outcomes. However, histological analysis of liver biopsies remains the standard against which other methods for assessment of the presence and amount of hepatic injury due to NAFLD are measured, especially for detecting NASH (hepatocyte ballooning and lobular inflammation) and how lobular inflammation differs from simple steatosis.

CAP could be used as a non-invasive, inexpensive, and accurate method to diagnose NAFLD in NAFLD risk groups (de Ledinghen 2014). Asia-Pacific guidelines propose CAP as a useful tool for diagnosis of NAFLD, as well as for demonstrating improvement in hepatic steatosis after lifestyle intervention and body-weight reduction (Chitturi 2018; Wong 2018), while other guidelines report that its role in clinical practice has yet to be determined (Chalasani 2018).

If the diagnostic accuracy of transient elastography plus CAP is shown to be better than the diagnostic accuracy of abdominal ultrasound, then it can replace abdominal ultrasound because transient elastography plus CAP is easier to interpret by the operator in view of the predefined parameters. Transient elastography plus CAP may replace liver biopsy, unless results of liver stiffness and grades of hepatic steatosis measurements are questionable (Eddowes 2019).

The downstream consequences of testing include the following.

- True-positive (TP): patients would benefit from rapid diagnosis and refer for an effective intervention.

- True-negative (TN): patients would be spared unnecessary treatment and would benefit from reassurance and pursuit of an alternative diagnosis.

- False-positive (FP): patients would probably experience anxiety and morbidity caused by additional testing, unnecessary treatment, and possible adverse events; and the chance that a FP result may halt further diagnostic evaluation.

- False-negative (FN): increased risk of morbidity and mortality and delayed treatment initiation, lifestyle modifications.

Figure 1 displays a summary of a currently used diagnostic pathway in assessing the grade of steatosis, choice of intervention, and monitoring and assessment of treatments. 
Figure 1. Clinical pathway. NAFLD: non-alcoholic fatty liver disease.

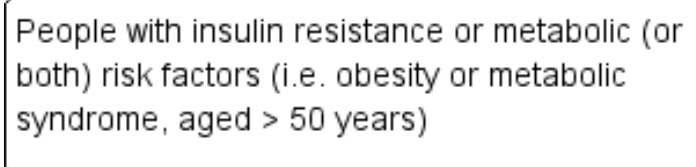

syndrome, aged $>50$ years)

People with persistently abnormal liver enzymes

People should undergo diagnostic

procedures for the diagnosis

NAFLD

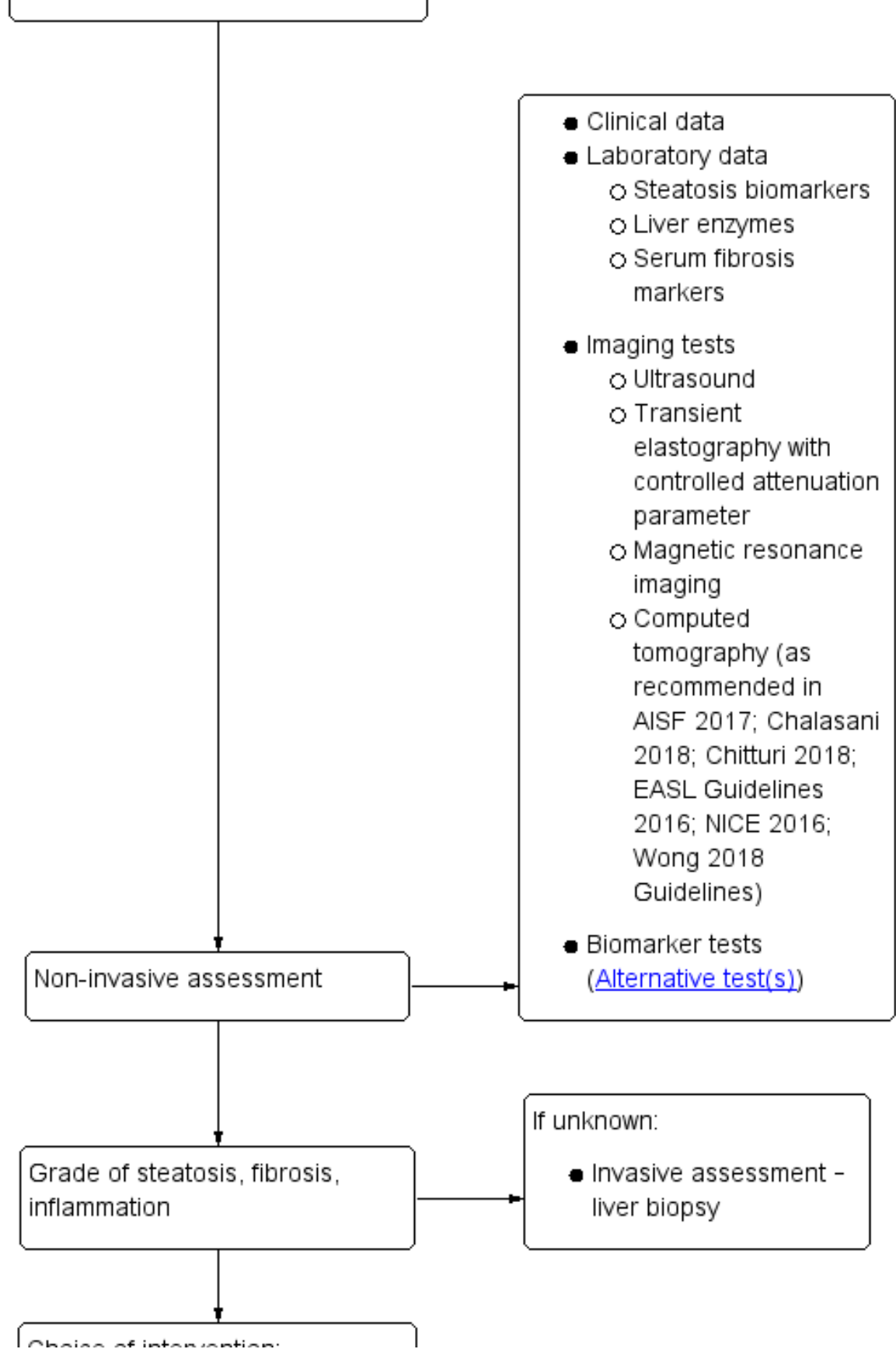

Transient elastography with controlled attenuation parameter (CAP) for diagnosis of moderate or severe steatosis in people with 
Figure 1. (Continued)

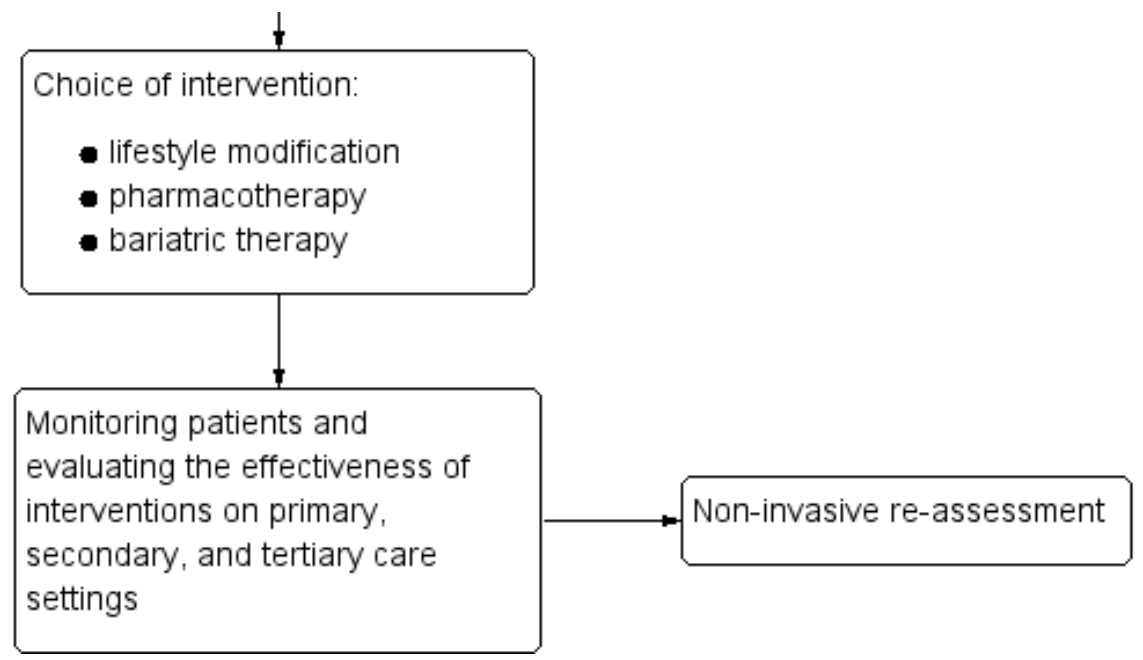

\section{Alternative test(s)}

\section{Biomarker tests}

FLI, NAFLD liver fat score (NAFLD-LFS), hepatic steatosis index (HSI), visceral adiposity index (VAI), and triglyceride-glucose (TyG) index are some of the biomarkers most often used to quantify steatosis. They are based on aggregate scores, and different anthropometric and metabolic parameters. However, their ability to quantify steatosis is low as shown by one meta-analysis by Fedchuk 2014. In addition, none of these biomarker tests can distinguish between moderate and severe steatosis (Fedchuk 2014). The areas under the receiver-operating-characteristic curves (AUROC) for predicting steatosis of more than $33 \%$ (i.e. moderate and severe) were 0.83 (95\% confidence interval $(\mathrm{Cl}) 0.72$ to 0.91$)$ for FLI, $0.80(95 \% \mathrm{Cl}$ 0.69 to 0.88$)$ for NAFLD-LFS, $0.81(95 \% \mathrm{Cl} 0.71$ to 0.88$)$ for HSI, $0.92(95 \% \mathrm{Cl} 0.85$ to 0.95$)$ for $\mathrm{VAI}$, and 0.90 (0.84 to 0.94$)$ for TyG indices (Fedchuk 2014). The patient data required to perform the tests are BMI, waist circumference, triglycerides, gamma-glutamyl transferase; presence or absence of metabolic syndrome or T2DM, insulin levels, AST, ALT, high-density lipoprotein cholesterol, and glucose (Fedchuk 2014).

\section{Other imaging tests}

Abdominal ultrasonography is the most common first-line imaging modality for people with elevated liver enzymes or suspected NAFLD. As compared to the right kidney parenchyma, distal attenuation, and the presence of areas of focal sparing, a typical ultrasonography feature of value to detect or exclude moderateto-pronounced fatty infiltration is hyperechogenicity (an increased response (echo) during the ultrasound examination) (Hernaez 2011). The degree of steatosis can be subjectively scored as mild, moderate, and severe, or as reported in some studies by using ordinal ultrasonography scores (Ballestri 2012). In one metaanalysis by Hernaez 2011, the pooled sensitivity and specificity of abdominal ultrasonography to distinguish moderate-to-severe fatty liver from the absence of steatosis, taking liver biopsy as the reference standard, were $84.8 \%(95 \% \mathrm{Cl} 79.5$ to 88.9$)$ (sensitivity) and $93.6 \%(95 \% \mathrm{Cl} 79.5$ to 88.9$)$ (specificity). Abdominal ultrasonography has limited sensitivity and does not reliably detect steatosis when it is less than $20 \%$ or in people with high BMI (greater than $40 \mathrm{~kg} / \mathrm{m}^{2}$ ) (EASL Guidelines 2016). It is also operatordependent.

Magnetic resonance-based methods are used for the detection or grading of hepatic steatosis and fibrosis. Diffusion-weighted magnetic resonance imaging (DW-MRI) measures the random Brownian motion of water molecules within a voxel of tissue. Magnetic resonance elastography (MRE) uses shear waves to assess the tissue displacement in all directions. Magnetic resonance spectroscopy (MRS) is based on the fact that the distribution of electrons within an atom causes nuclei in different molecules to experience slightly different magnetic fields (Tognarelli 2015). DWMRI, MRE, and MRS have an overall AUROC of 0.90 , sensitivity of $82 \%$, and specificity of $87 \%$. They also exhibit good AUROC (DWMRI 0.95 ( $95 \% \mathrm{Cl} 0.93$ to 0.97 ); MRE 0.89 (95\% Cl 0.86 and 0.91), and MRS 0.88 ( $95 \% \mathrm{Cl} 0.82$ to 0.93 ) (Wang 2018). Magnetic resonance imaging proton density fat fraction (MRI-PDFF) of liver tissue is an imaging biomarker derived from the fat and water component images acquired during an MRI examination and computed as the ratio of the fraction of mobile protons belonging to triglyceride, relative to those to water. According to Gu 2019, the summary AUROC values of MRI-PDFF for classifying steatosis grades 0 versus 1 to 3 are $0.98,0$ to 1 versus 2 to 3 are 0.91 , and 0 to 2 versus 3 are 0.90 . All these methods are accurate and fast methods of detecting liver fat, allowing detailed examination of areas of interest, but they are expensive and may not be routinely available (Wang 2018; Gu 2019).

Computed tomography can measure tissue density in Hounsfield units (HU) from air $(-1000)$ at the bottom of the scale, to bone density at the top of the scale. Computed tomography diagnostic criteria for steatosis are liver attenuation, at least 10 Hounsfield Units (HU) less than that of the spleen (sensitivity $60.5 \%$ and specificity $100 \%$ ), or absolute liver attenuation of less than $40 \mathrm{HU}$ (sensitivity $52.5 \%$ and specificity $100 \%$ ) (Lawrence 2012).

\section{Rationale}

Steatosis generally has a favourable outcome, although people with isolated hepatic steatosis and with clinical diagnosis of metabolic syndrome or individual components of metabolic syndrome may be at risk of more rapidly progressive histological inflammatory damage and adverse outcomes associated with

Transient elastography with controlled attenuation parameter (CAP) for diagnosis of moderate or severe steatosis in people with 
NASH (Pais 2013; Rinella 2016). Some studies suggest that the degree of steatosis may predict the severity of histological features (Chalasani 2008), and the incidence and prevalence of diabetes in people with NAFLD (Li 2013; Shah 2015; Targher 2016). The aggregate data provide strong evidence that people with NAFLD are at increased 'independent' risk of developing cardiovascular disease (Armstrong 2014). Similarly, the bidirectional association between NAFLD and components of metabolic syndrome has been strongly established. Also, there are established conditions such as obesity, T2DM, hypertension, and dyslipidaemia, and emerging conditions, such as sleep apnoea, colorectal cancer, osteoporosis, psoriasis, endocrinopathies, and polycystic ovary syndrome independent of obesity, that are associated with NAFLD (Chalasani 2018). The European Association for the Study of the Liver recommends diagnosing obese people, people with a metabolic syndrome, and people at high risk of developing a cardiovascular disease for presence of NAFLD (EASL Guidelines 2016). The ideal method for evaluation of liver steatosis in people with suspected NAFLD must be widely available, non-invasive, safe, sensitive, accurate for quantification, reproducible, and inexpensive.

The meta-analyses by Shi 2014 and Wang 2015 showed a high sensitivity and specificity of CAP for NAFLD of S1 or greater, S2 or greater, and S3 or greater (Table 2). However, the study authors did not recommend using this method to diagnose liver steatosis in routine clinical practice. Meta-analyses conducted by Karlas 2017 and Pu 2019 showed high diagnostic accuracy of transient elastography with CAP. However, the Pu 2019 metaanalysis has limitations regarding databases searched and have flaws in methodology, including lack of risk of bias assessment. The meta-analysis of individual data by Karlas 2017 included 537 participants, and the meta-analysis by Pu 2019 included 1297 participants. However, there are other relevant studies, not included in the mentioned meta-analyses.

As researchers are using different cut-off values to determine the stage of steatosis in people with NAFLD, we consider it necessary to conduct this Cochrane systematic review on the diagnostic accuracy of transient elastography with CAP for diagnosis of moderate and severe steatosis in people with suspected NAFLD, including the available evidence.

\section{O B J E C T IVES}

To determine the diagnostic accuracy of transient elastography with CAP for diagnosis of moderate and severe hepatic steatosis in people with suspected NAFLD when compared with liver biopsy as reference standard. To achieve this, we will compare none/ mild hepatic steatosis (S0 to S1) versus moderate/severe hepatic steatosis (S2 to S3); and none/mild/moderate hepatic steatosis (S0 to S2) versus severe hepatic steatosis (S3).

\section{Secondary objectives}

We aim to also identify the pooled sensitivity and specificity for the most common cut-off values of CAP for diagnosis of none/mild hepatic steatosis ( $\mathrm{S} 0$ to $\mathrm{S} 1$ ) and moderate/severe hepatic steatosis (S2 to S3); or none/mild/moderate hepatic steatosis (S0 to S2) and severe hepatic steatosis (S3) in people with suspected NAFLD, and to explore potential sources of heterogeneity influencing the diagnostic test accuracy of CAP (see: Investigations of heterogeneity).

\section{METHODS}

\section{Criteria for considering studies for this review}

\section{Types of studies}

We will include cross-sectional studies with liver histology as reference standard and transient elastography with CAP as index test, providing diagnostic accuracy data (TP, FP, FN, and TN) in people with suspected NAFLD. We will include studies irrespective of publication status, format, and language. We will exclude studies of case-control design that compared people with known NAFLD to matched controls. Case-control designs are considered at high risk of bias, as they inflate accuracy estimates (Colli 2014).

\section{Participants}

Study participants can be hospitalised or outpatients, of any sex and ethnic origin, and at least 18 years old, with suspected NAFLD. People may present with any diabetes status, and both secondary causes and a daily alcohol consumption, defined as more than $30 \mathrm{~g} /$ day for men and more than $20 \mathrm{~g} /$ day for women, should be excluded. We will exclude studies with participants who had previously undergone liver transplantation due to a problem with the accuracy verifying the aetiology of steatosis. Majority of people with a long-term survival after liver transplantation have allografts that are histologically abnormal. This may be caused by the effects of immunosuppression or interactions with other graft complications (Hubscher 2011).

\section{Index tests}

\section{Transient elastography with controlled attenuation parameter}

Most often, authors use various cut-off values to grade liver steatosis: a cut-off value of $259.4 \mathrm{~dB} / \mathrm{m}$ to $268 \mathrm{~dB} / \mathrm{m}$ for S0/S1 versus $\mathrm{S} 2 / \mathrm{S} 3$, and a cut-of value of $280 \mathrm{~dB} / \mathrm{m}$ to $292.3 \mathrm{~dB} / \mathrm{m}$ for S0/S2 versus S3 (Sasso 2010; Karlas 2017). For our review, we will consider the cut-off values of transient elastography with CAP as used by the study authors.

\section{Target conditions}

The target conditions will be moderate or severe hepatic steatosis in people with suspected NAFLD according histopathological scoring systems for NAFLD (Table 1).

\section{Reference standards}

Liver histology with assessment of steatosis.

A liver biopsy allows confirmation of the presence of NAFLD, to differentiate diagnosis between steatosis and steatohepatitis, to assess the stage of fibrosis, and, based on histological data, to predict the development of the disease as well as to exclude other causes of liver damage. However, liver biopsy is not practical when a disease and its progression needs to be monitored. It is an invasive procedure and carries a small, but definite risk of complications. It is also unrealistic to perform liver biopsy in $15 \%$ to $40 \%$ of the general population.

Following histological scoring systems, there are four grades of hepatic steatosis: minimal steatosis (grade 0), defined as presence of less than $5 \%$ or $10 \%$; mild steatosis, between $5 \%$ and $33 \%$ (grade 1); moderate steatosis, between $33 \%$ and $66 \%$ (grade 2); and severe steatosis, more than $66 \%$ (grade 3 ). The steatohepatitis 
elements are steatosis complicated by liver cell injury (evident as ballooned hepatocytes, or Mallory hyaline) with substantial lobular (and occasionally portal) inflammation. The histological changes characterising the stages of fibrosis are defined as stage 1 (perisinusoidal fibrosis); stage 2 (perisinusoidal fibrosis with periportal fibrosis); stage 3 (bridging fibrosis); and stage 4 (fully developed cirrhosis) (Brunt 1999; Kleiner 2012; Bedossa 2014).

Different histopathological scoring systems are used to diagnose or predict progression of NAFLD (Table 1). The NAFLD activity score (NAS) evaluates the degree of morphological changes in scores (Kleiner 2005). It is a modification of the Brunt and Matteoni scales used earlier in 1999 (Brunt 1999). The SAF (steatosis, activity, fibrosis) semi-quantitative score, proposed by Bedossa 2014 , assesses the severity of NAFLD based on severity of steatosis (S, steatosis), balloon dystrophy and lobular inflammation ( $\mathrm{A}$ activity), and the stage of liver fibrosis ( $F$, fibrosis). The results of the SAF score assessments are used as an index for NASH staging (e.g. S1A2F3, S2A1F1, etc.) (Bedossa 2014). We will use a conversion grid for hepatic fibrosis staging if a study assesses fibrosis according to Metavir, Kleiner, or Ishak score (French METAVIR CSG 1994: Ishak 1995; Kleiner 2005; Table 4). We will add other histopathological scoring systems to Table 4 if such are used in the included studies.

\section{Search methods for identification of studies}

We will combine electronic searches with reading references of the identified studies of possible interest. We will include all studies irrespective of language of publication and publication status. We will translate non-English language articles with the help of Cochrane Hepato-Biliary Group (CHBG) collaborators.

\section{Electronic searches}

We will search The Cochrane Hepato-Biliary Group Controlled Trials Register (maintained and searched internally by the CHBG Information Specialist via the Cochrane Register of Studies Web), the Cochrane Hepato-Biliary Group Diagnostic Test Accuracy Studies Register (maintained and searched internally by the CHBG Information Specialist via the Cochrane Register of Studies Web), the Cochrane Central Register of Controlled Trials (CENTRAL), MEDLINE Ovid, Embase Ovid), CINAHL (EBSCOhost), LILACS (Bireme), Science Citation Index Expanded (Web of Science), and Conference Proceedings Citation Index - Science (Web of Science) (Royle 2003). We will also search ClinicalTrial.gov (clinicaltrials.gov/), European Medicines Agency (EMA) (www.ema.europa.eu/ema/), World Health Organization International Clinical Trial Registry Platform (www.who.int/ictrp), and the Food and Drug Administration (FDA) (www.fda.gov).

Appendix 1 shows the preliminary search strategies for the different databases with the expected time spans for the searches. The given search strategies may be improved at the review preparation phase.

\section{Searching other resources}

We will search the references of the included studies to identify additional studies. We will also search for articles related to the included studies by performing the 'related search' function in MEDLINE (OvidSP) and Embase (OvidSP) and a 'citing reference' search (by searching the articles that cite the included articles) in these databases. We will also search for other types of grey literature in the System for Information on Grey Literature in Europe OpenGrey (www.opengrey.eu/).

\section{Data collection and analysis}

We will follow available guidelines as provided in the Cochrane Handbook for Diagnostic Test of Accuracy Reviews (DTA Handbook 2013).

\section{Selection of studies}

Three review authors ( $\mathrm{TT}, \mathrm{AB}$, and $\mathrm{CP}$ ) will independently identify relevant studies from the retrieved references. The first selection will be based on reference title or abstract. For the second selection, we will retrieve the full texts of selected studies considered to be relevant by at least one of the review authors. Two review authors will independently screen the full-text of the publications against the inclusion criteria and will resolve any differences through discussion. We will remove duplicates and list multiple publications of the same study within its main reference. We will record the number and details of all studies not fulfilling the inclusion criteria, based on full-text assessment in the 'Characteristics of excluded studies' table, with the reasons for exclusion. We will illustrate the study selection process using a PRISMA diagram (Moher 2011). We will solve disagreements through discussion. If disagreements persist, AS or KSG will serve as arbitrator.

\section{Data extraction and management}

Three review authors ( $\mathrm{TT}, \mathrm{AB}$, and $\mathrm{CP}$ ) will independently extract data from each included study, using a prepiloted data extraction form by TT (Table 5). We will then compare our data extraction, and we will resolve differences by discussion.

We will extract all necessary data to calculate TP, FP, TN, and FN values, using the reference standard of liver histology. We will summarise the data from each study in $2 \times 2$ tables (TP, FP, FN, TN), according to the index tests considered. We will use Review Manager 5 software to enter data and perform analyses (Review Manager 2014). If information on any of the TP, FP, FN, and TN diagnostic test values or results are missing, we will attempt to derive these using other information that the study may provide.

\section{Missing data}

We will contact primary authors of abstract publications and fulltext publications by e-mail to ask for missing data which are needed to design the $2 \times 2$ tables. If we receive no reply, we will send a second e-mail after two weeks. If no reply is received, then we will list the study in 'Excluded studies', with a reason for exclusion.

In case a full-text publication of a study, presented only in an abstract format, is not found, and the abstract lacks the data we need, and study authors do not respond to our requests for missing data, we will judge if we shall list the abstract publication within 'Excluded studies' or 'Studies awaiting assessment', in view of future update of the review.

\section{Assessment of methodological quality}

We will use the QUADAS-2 (QUality Assessment of Diagnostic Accuracy Studies) tool, to assess risk of bias in diagnostic test accuracy studies and the applicability of their results. QUADAS-2 is based on four quality domains: participant selection, index test, reference standard, and flow and timing (Whiting 2011; QUADAS-2). There are signalling questions for each of the domains, used to make judgements in terms of risk of bias. The answers to the signalling questions are 'yes', 'no', or 'unclear'. The first 
three domains are also judged in terms of concerns regarding applicability, and the answers can be 'low', 'high', or 'unclear'.

Appendix 2 shows the signalling questions to our review subject, and the criteria that we will use to assess the methodological quality of each study in our review. Regarding the 'flow and timing' domain, we will consider an interval of three months (12 weeks) the most appropriate interval between the reference standard test and the index test. However, in studies where participants underwent bariatric surgery, we will consider an interval of one month (four weeks) the most appropriate interval between the reference standard test and the index test. This is due to data that have shown a significant decrease in steatosis three months after bariatric surgery (Aldoheyan 2017).

Two review authors (TT and $A B$ ) will independently judge risk of bias in each domain and applicability of results. They will provide quotes and comments to support each judgement for each domain. In case of discrepancies and no agreement reached, a third author (CP or KSG) will act as arbitrator.

If we judge a study as 'low' on all domains relating to bias or applicability, then our overall judgement for that study will be 'low risk of bias' or 'low concern regarding applicability'. If we judge a study as 'high' or 'unclear' in one or more domains relating to bias or applicability, then our overall judgement for that study will be 'at risk of bias' or as having 'concerns regarding applicability'.

The guidance to arrive at judgements for risk of bias in each study domain is provided in Appendix 2.

Two review authors (TT and AB) will present the results in 'Risk of bias' summaries and graphs in addition to a narrative summary, as advised on the QUADAS-2 website. We will check the QUADAS-2 website for possible updates during the review preparation.

\section{Statistical analysis and data synthesis}

We will carry out statistical analyses according to recommendations provided in the Cochrane Handbook for Systematic Reviews of Diagnostic Test Accuracy (Macaskill 2010; DTA Handbook 2013). The analyses will be performed using Review Manager 5 (Review Manager 2014) and Stata Statistical Software (Release 16. TX: StataCorp LLC).

We will build $2 \times 2$ tables of CAP performance (TP, TN, FP, FN) for all of the predefined target conditions (moderate and severe steatosis). We will estimate sensitivity, specificity, and positive and negative likelihood ratios (LR+ and LR-) with their 95\% Cls. Summary LR+ and LR- will be calculated as part of the METADAS code using the formula LR+ = sensitivity/(1 - specificity $)$ and LR- = (1 - specificity)/sensitivity (DTA Handbook 2013). First, we will perform a graphical descriptive analysis of the included studies: we will present coupled forest plots of sensitivity and specificity separately, with their $95 \% \mathrm{Cls}$, and we will provide a graphical representation of the studies in the receiver operating characteristic (ROC) space (sensitivity against 1 - specificity).

Second, where appropriate, we will perform meta-analyses. The method that we will use to estimate summary statistic will depend on the variability in positivity thresholds. If the primary studies report accuracy estimates of CAP using different cut-off values, we will use the hierarchical summary ROC model (HSROC) to pool data (sensitivities and specificities) and to estimate the summary ROC (SROC) curve (Macaskill 2010).

If performance reported across the primary studies is based on consistent cut-off values, we will use the bivariate model, and we will provide estimates of summary sensitivity and specificity.

For primary studies that report accuracy results for more than one cut-off value, we will report sensitivities and specificities for all of the cut-off values, but we will use a single cut-off value of CAP for each study in HSROC analysis. This value will be the most frequently used cut-off value.

We do not plan to include non-evaluable index test results in our main statistical analysis. We plan to perform an 'intentionto-diagnose' analysis as an additional sensitivity analysis (See Sensitivity analyses). We will classify participants with nonevaluable index test results as FPs if they had a negative reference standard or FNs with a positive reference standard (Schuetz 2012). We will use $3 \times 2$ tables. If data for the intention-to-diagnose analyses are not retrievable from the text, and as mentioned earlier, we will contact publication authors. If we receive no reply, we will include the study in the analyses with data retrievable from the published manuscript.

We will rate the certainty of the evidence, and we will present a summary of our findings, using the GRADE approach (GRADE Handbook 2013; GRADEpro GDT) (detailed below).

\section{Investigations of heterogeneity}

We plan to investigate if the following potential sources of heterogeneity may influence our overall results.

- As there is a significant variation in the activity of steatohepatitis and liver fibrosis among patients, we will analyse:

* the different levels of histological activity (from 0 to 4): studies including more than $50 \%$ of participants with the sum of ballooning and lobular inflammation below 2 compared to studies including more than $50 \%$ of participants with 2 or greater grades of activity - categorical covariate;

* the different serum levels of ALT activity (increased ALT activity will be defined as serum ALT above the upper limit of normal, that is, more than $40 \mathrm{IU} / \mathrm{L}$ (Gawrieh 2019)): studies including more than $50 \%$ of participants with normal serum levels of ALT compared to studies including more than $50 \%$ of participants with serum levels of ALT greater than the upper limit - categorical covariate;

* the different levels of liver stiffness, in kilopascals (studies including more than $50 \%$ of participants with F0/F1/F2 with a cut-off $8.8 \mathrm{kPa}$ compared to studies including more than $50 \%$ of participants with F2/F3 with a cut-off $12.0 \mathrm{kPa}$ compared to studies including more than $50 \%$ of participants with F3/F4 with a cut-off $38.6 \mathrm{kPa}$ (Boursier 2016)) - categorical covariate;

* the different levels of histological fibrosis stage (studies including more than $50 \%$ of participants with F0/F1/F2 compared to studies including more than $50 \%$ of participants with F3/F4 (Table 4)) - categorical covariate. 
- As T2DM is associated with the development of NAFLD and can lead to advanced stages, we will analyse:

* studies including more than $50 \%$ of participants with T2DM compared to studies including more than $50 \%$ of participants without T2DM (a categorical covariate).

- As the presence and severity of obesity may limit the obtaining of reliable CAP measurements and requires the use of XL probe, we will analyse:

* the different BMIs; studies including more than $50 \%$ of participants with BMI below $25 \mathrm{~kg} / \mathrm{m}^{2}$ compared to studies including more than $50 \%$ of participants with BMI $25 \mathrm{~kg} / \mathrm{m}^{2}$ or more) (a categorical covariate);

* the different probes for measuring CAP: studies including more than $50 \%$ of participants with $\mathrm{M}$ probe or studies including more than $50 \%$ of participants with XL probe (a categorical covariate).

- As there are differences in epidemiology of NAFLD in continents and subcontinents, we will analyse:

* the study location (population differences): European compared to Asia compared to Africa compared to America (Northern America compared to South America, if relevant studies will be found) (a categorical covariate).

We will estimate effects by adding covariates to the bivariate or HSROC model (depending on the model chosen based on the variation in positivity thresholds reported in the studies). We will assess the statistical significance of the covariate effect using the log-likelihood ratio test for comparison of models with and without the covariate term. We will consider a P less than 0.05 as two-sided and statistically significant.

\section{Sensitivity analyses}

We will perform sensitivity analysis by excluding studies:

- at high risk of bias, that is, studies having high risk of bias in at least one of the domains of QUADAS-2 (Appendix 2);

- published only in abstract or letter form;

- with 'no' or 'unclear' answer to the signalling questions: "If a threshold was used, was it prespecified?", "Was there an appropriate interval between the index test and the reference standard?"

- with 'no' or 'unclear' answer to the signalling question: "Was there an appropriate interval between the index test and the reference standard?"

- intention-to-diagnose analyses in terms of index test results (Schuetz 2012).

Publication bias: we will rate publication bias as undetected (not serious) because of the comprehensiveness of the literature search and following extensive outreach to researchers to identify studies.

\section{Certainty of the evidence and 'Summary of findings' table}

We will consider the following GRADE factors to assess the certainty of the evidence:

- risk of bias: we will use QUADAS-2 to assess risk of bias;

- indirectness: for the review questions, we will look for important differences between the populations studied (e.g. the spectrum of disease), the setting, the index test, and the reference standard;

- inconsistency: we will carry out prespecified analyses to investigate potential sources of heterogeneity, and we will downgrade the evidence when we cannot explain inconsistency in the accuracy estimates;

- imprecision: we will look at the Cls of sensitivity and specificity estimates and at the unexplained heterogeneity of the results; or TP, FP, FN, and TN rates;

- publication bias: we will use the trim-and-fill method (Schünemann 2020).

We will follow the instructions in Section 7.5. 'Judgment about the quality of the underlying evidence' of the GRADE Handbook 2013 on how to downgrade or upgrade the evidence. We will justify all our decisions, using footnotes, and we will make comments to aid reader's understanding of the review, where necessary.

We will prepare a 'Summary of Findings' table to present the main results and key information regarding our assessment of the certainty of evidence, using the GRADE approach (GRADE Handbook 2013; GRADEpro GDT).

We will rate the certainty of the evidence in one of the four grades: high, moderate, low, or very low, defined as follows (GRADE Handbook 2013; GRADEpro GDT).

- High: we are very confident that the true effect lies close to that of the effect estimate.

- Moderate: we are moderately confident of the effect estimate and that the true effect is likely to be close to the effect estimate, but there is a possibility that it is substantially different.

- Low: our confidence in the effect estimate is limited, such that the true effect may be substantially different from the effect estimate.

- Very low: we have very little confidence in the effect estimate, such that the true effect is likely to be substantially different from the estimate of effect.

\section{ACK N O WLEDGEMENTS}

Cochrane Review Group funding acknowledgement: the Danish State is the largest single funder of The Cochrane Hepato-Biliary Group through its investment in The Copenhagen Trial Unit, Centre for Clinical Intervention Research, Rigshospitalet, Copenhagen University Hospital, Denmark. Disclaimer: the views and opinions expressed in this review are those of the authors and do not necessarily reflect those of the Danish State or The Copenhagen Trial Unit.

The authors would like to thank the Russian Foundation for Basic Research (RFBR), Russia, (grant RFBR No. 19-54-18011\19) for help in identifying current issues that need to be addressed in people with liver cirrhosis in Russia and Bulgaria.

Peer reviewers: Daniele Prati, Italy.

Contact Editor from The Cochrane Diagnostic Accuracy Reviews Editorial Team: Karen R Steingart, UK.

Contact editor: Agostino Colli, Italy.

Sign-off editor: Christian Gluud, Denmark. 


\section{RE F E R E N C E S}

\section{Additional references}

\section{Adams 2005}

Adams LA, Lymp JF, St Sauver J, Sanderson SO, Lindor KD, Feldstein A, et al. The natural history of nonalcoholic fatty liver disease: a population-based cohort study. Gastroenterology 2005;129(1):113-21. [PMID: 16012941]

\section{AISF 2017}

Italian Association for the Study of the Liver. AISF position paper on nonalcoholic fatty liver disease (NAFLD): updates and future directions. Digestive and Liver Disease 2017;49(5):471-83. [PMID: 28215516]

\section{Aldoheyan 2017}

Aldoheyan T, Hassanain M, Al-Mulhim A, Al-Sabhan A, AlAmro S, Bamehriz F, et al. The effects of bariatric surgeries on nonalcoholic fatty liver disease. Surgical Endoscopy 2017;31(3):1142-7. [PMID: 27405478]

\section{Angulo 2002}

Angulo P. Nonalcoholic fatty liver disease. New England Journal of Medicine 2002;346(16):1221-31. [PMID: 11961152]

\section{Angulo 2013}

Angulo P. The natural history of NAFLD. In: Farrell GC, McCullough AJ, Day CP, editors(s). Non-Alcoholic Fatty Liver Disease: a Practical Guide. Hoboken (NJ): John Wiley \& Sons, 2013:37-45.

\section{Angulo 2015}

Angulo P, Kleiner DE, Dam-Larsen S, Adams LA, Bjornsson ES, Charatcharoenwitthaya $P$, et al. Liver fibrosis, but no other histologic features, is associated with long-term outcomes of patients with nonalcoholic fatty liver disease. Gastroenterology 2015;149(2):389-97.e10. [PMID: 25935633]

\section{Armstrong 2014}

Armstrong MJ, Adams LA, Canbay A, Syn WK. Extrahepatic complications of nonalcoholic fatty liver disease. Hepatology (Baltimore, Md.) 2014;59(3):1174-97. [PMID: 24002776]

\section{Ballestri 2012}

Ballestri S, Lonardo A, Romagnoli D, Carulli L, Losi L, Day CP, et al. Ultrasonographic fatty liver indicator, a novel score which rules out NASH and is correlated with metabolic parameters in NAFLD. Liver International 2012;32(8):1242-52. [PMID: 22520641]

\section{Bedossa 2014}

Bedossa P. Utility and appropriateness of the fatty liver inhibition of progression (FLIP) algorithm and steatosis, activity, and fibrosis (SAF) score in the evaluation of biopsies of nonalcoholic fatty liver disease. Hepatology (Baltimore, Md.) 2014;60(2):565-75. [PMID: 24753132]

\section{Boursier 2016}

Boursier J, Vergniol J, Guillet A, Hiriart JB, Lannes A, Le Bail B, et al. Diagnostic accuracy and prognostic significance of blood fibrosis tests and liver stiffness measurement by FibroScan in non-alcoholic fatty liver disease. Journal of Hepatology 2016;65(3):570-8. [PMID: 27151181]

\section{Brunt 1999}

Brunt EM, Janney CG, Di Bisceglie AM, Neuschwander-Tetri BA, Bacon BR. Nonalcoholic steatohepatitis: a proposal for grading and staging the histological lesions. American Journal of Gastroenterology 1999;94(9):2467-74. [PMID: 10484010]

\section{Castera 2010}

Castera L, Foucher J, Bernard PH, Carvalho F, Allaix D, Merrouche W, et al. Pitfalls of liver stiffness measurement: a 5 -year prospective study of 13,369 examinations. Hepatology (Baltimore, Md.) 2010;51(3):828-35. [PMID: 20063276]

\section{Chalasani 2008}

Chalasani N, Wilson L, Kleiner DE, Cummings OW, Brunt EM, Unalp A. Relationship of steatosis grade and zonal location to histological features of steatohepatitis in adult patients with non-alcoholic fatty liver disease. Journal of Hepatology 2008;48(5):829-34. [PMID: 18321606]

\section{Chalasani 2012}

Chalasani N, Younossi Z, Lavine JE, Diehl AM, Brunt EM, Cusi K, et al. The diagnosis and management of non-alcoholic fatty liver disease: practice guideline by the American Gastroenterological Association, American Association for the Study of Liver Diseases, and American College of Gastroenterology. Gastroenterology 2012;142(7):1592-609. [PMID: 22656328]

\section{Chalasani 2018}

Chalasani N, Younossi Z, Lavine JE, Charlton M, Cusi K, Rinella $M$, et al. The diagnosis and management of nonalcoholic fatty liver disease: practice guidance from the American Association for the Study of Liver Diseases. Hepatology (Baltimore, Md.) 2018;67(1):328-57. [PMID: 28714183]

\section{Chitturi 2018}

Chitturi S, Wong VW, Chan WK, Wong GL, Wong SK, Sollano J, et al. The Asia-Pacific Working Party on Non-alcoholic Fatty Liver Disease guidelines 2017-Part 2: management and special groups. Journal of Gastroenterology and Hepatology 2018;33(1):86-98. [PMID: 28692197]

\section{Colli 2014}

Colli A, Fraquelli M, Casazza G, Conte D, Nikolova D, Duca P, et al. The architecture of diagnostic research: from bench to bedside - research guidelines using liver stiffness as an example. Hepatology (Baltimore, Md.) 2014;60(1):408-18. [PMID: 24277656]

\section{de Ledinghen 2010}

de Ledinghen V, Vergniol J, Foucher J, El-Hajbi F, Merrouche W, Rigalleau V. Feasibility of liver transient elastography with FibroScan using a new probe for obese patients. Liver International 2010;30(7):1043-8. [PMID: 20492500] 


\section{de Ledinghen 2014}

de Ledinghen V, Vergniol J, Capdepont M, Chermak F, Hiriart $\mathrm{J}-\mathrm{B}$, Cassinotto $\mathrm{C}$, et al. Controlled attenuation parameter (CAP) for the diagnosis of steatosis: a prospective study of 5323 examinations. Journal of Hepatology 2014;60(5):1026-31.

\section{DTA Handbook 2013}

Deeks JJ, Bossuyt PM, Gatsonis C, editor(s). Cochrane Handbook for Systematic Reviews of Diagnostic Test Accuracy Version 1.0.0. The Cochrane Collaboration, 2013. Available from srdta.cochrane.org.

\section{EASL Guidelines 2016}

European Association for the Study of the Liver (EASL), European Association for the Study of Diabetes (EASD), European Association for the Study of Obesity (EASO). EASLEASD-EASO clinical practice guidelines for the management of non-alcoholic fatty liver disease. Journal of Hepatology 2016;64(6):1388-402. [PMID: 27062661]

\section{Echosens 2011}

Echosens. FibroScan 502. Innovation in liver disease management. delruseurope.com/wp-content/uploads/ BrochureFS502TouchEN-WEB.compressed.pdf 2011 (accessed 11 June 2019).

\section{Eddowes 2019}

Eddowes PJ, Sasso M, Allison M, Tsochatzis E, Anstee QM, Sheridan D, et al. Accuracy of FibroScan controlled attenuation parameter and liver stiffness measurement in assessing steatosis and fibrosis in patients with nonalcoholic fatty liver disease. Gastroenterology 2019;156(6):1717-30. [PMID: 30689971]

\section{Ekstedt 2006}

Ekstedt M, Franzen LE, Mathiesen UL, Thorelius L, Holmqvist M, Bodemar G, et al. Long-term follow-up of patients with NAFLD and elevated liver enzymes. Hepatology (Baltimore, Md.) 2006;44(4):865-73. [PMID: 17006923]

\section{Ekstedt 2015}

Ekstedt M, Hagstrom H, Nasr P, Fredrikson M, Stal P, Kechagias $\mathrm{S}$, et al. Fibrosis stage is the strongest predictor for disease-specific mortality in NAFLD after up to 33 years of follow-up. Hepatology (Baltimore, Md.) 2015;61(5):1547-54. [PMID: 25125077]

\section{Fedchuk 2014}

Fedchuk L, Nascimbeni F, Pais R, Charlotte F, Housset C, Ratziu V. Performance and limitations of steatosis biomarkers in patients with nonalcoholic fatty liver disease. Alimentary Pharmacology \& Therapeutics 2014;40(10):1209-22. [PMID: 25267215]

\section{French METAVIR CSG 1994}

French METAVIR Cooperative Study Group. Intraobserver and interobserver variations in liver biopsy interpretation in patients with chronic hepatitis C. Hepatology (Baltimore, Md.) 1994;20(1 Pt 1):15-20. [PMID: 8020885]

\section{Gawrieh 2019}

Gawrieh S, Wilson LA, Cummings OW, Clark JM, Loomba R, Hameed B, et al. Histologic findings of advanced fibrosis and cirrhosis in patients with nonalcoholic fatty liver disease who have normal aminotransferase levels. American Journal of Gastroenterology 2019;114(10):1626-35. [PMID: 31517638]

\section{GRADE Handbook 2013}

Schünemann H, Brożek J, Guyatt G, Oxman A, editor(s). Handbook for grading the quality of evidence and the strength of recommendations using the GRADE approach (updated October 2013). GRADE Working Group, 2013. Available from gdt.guidelinedevelopment.org/app/handbook/handbook.html.

\section{GRADEpro GDT [Computer program]}

McMaster University (developed by Evidence Prime) GRADEpro GDT. Version accessed 29 June 2020. Hamilton (ON): McMaster University (developed by Evidence Prime), 2015. Available at gradepro.org.

\section{Gu 2019}

Gu J, Liu S, Du S, Zhang Q, Xiao J, Dong Q, et al. Diagnostic value of MRI-PDFF for hepatic steatosis in patients with non-alcoholic fatty liver disease: a meta-analysis. European Radiology 2019;29(7):3564-73. [PMID: 30899974]

\section{Gurusamy 2018}

Gurusamy KS, Tsochatzis E, Madden AM. Lifestyle modifications for non-alcohol related fatty liver disease: a network metaanalysis. Cochrane Database of Systematic Reviews 2018, Issue 10. Art. No: CD013156. [DOI: 10.1002/14651858.CD013156] [CD013156]

\section{Hernaez 2011}

Hernaez R, Lazo M, Bonekamp S, Kamel I, Brancati FL, Guallar E, et al. Diagnostic accuracy and reliability of ultrasonography for the detection of fatty liver: a meta-analysis. Hepatology (Baltimore, Md.) 2011;54(3):1082-90. [PMID: 21618575]

\section{Hubscher 2011}

Hubscher SG. What is the long-term outcome of the liver allograft? Journal of Hepatology 2011;55(3):702-17. [PMID: 21426919]

\section{Ishak 1995}

Ishak K, Baptista A, Bianchi L, Callea F, De Groote J, Gudat F, et al. Histological grading and staging of chronic hepatitis. Journal of Hepatology 1995;22(6):696-9. [PMID: 7560864]

\section{Karlas 2017}

Karlas T, Petroff D, Sasso M, Fan JG, Mi YQ, de Ledinghen V, et al. Individual patient data meta-analysis of controlled attenuation parameter (CAP) technology for assessing steatosis. Journal of Hepatology 2017;66(5):1022-30. [PMID: 28039099]

\section{Kleiner 2005}

Kleiner DE, Brunt EM, Van Natta M, Behling C, Contos MJ, Cummings OW, et al. Design and validation of a histological scoring system for nonalcoholic fatty liver disease. Hepatology (Baltimore, Md.) 2005;41(6):1313-21. [PMID: 15915461] 


\section{Kleiner 2012}

Kleiner DE, Brunt EM. Nonalcoholic fatty liver disease: pathologic patterns and biopsy evaluation in clinical research. Seminars in Liver Disease 2012;32(1):3-13. [PMID: 22418883]

\section{Kwak 2015}

Kwak M-S, Kim D. Long-term outcomes of nonalcoholic fatty liver disease. Current Hepatology Reports 2015;14(2):69-76.

\section{Lawrence 2012}

Lawrence DA, Oliva IB, Israel GM. Detection of hepatic steatosis on contrast-enhanced CT images: diagnostic accuracy of identification of areas of presumed focal fatty sparing. American Journal of Roentgenology 2012;199(1):44-7.

\section{Leoni 2018}

Leoni S, Tovoli F, Napoli L, Serio I, Ferri S, Bolondi L. Current guidelines for the management of non-alcoholic fatty liver disease: a systematic review with comparative analysis. World Journal of Gastroenterology 2018;24(30):3361-73. [PMID: 30122876]

\section{Li 2013}

Li X, Xia M, Ma H, Hu Y, Yan H, He W, et al. Liver fat content, evaluated through semi-quantitative ultrasound measurement, is associated with impaired glucose profiles: a communitybased study in Chinese. PloS One 2013;8(7):e65210. [PMID: 23843938]

\section{Lombardi 2017}

Lombardi R, Onali S, Thorburn D, Davidson BR, Gurusamy KS, Tsochatzis E. Pharmacological interventions for non-alcohol related fatty liver disease (NAFLD). Cochrane Database of Systematic Reviews 2017, Issue 3. Art. No: CD011640. [DOI: 10.1002/14651858.CD011640.pub2] [CD011640]

\section{Macaskill 2010}

Macaskill P, Gatsonis C, Deeks JJ, Harbord RM, Takwoingi Y. Chapter 10: Analysing and presenting results. In: Deeks JJ, Bossuyt PM, Gatsonis, C, editor(s), Cochrane Handbook for Systematic Reviews of Diagnostic Test Accuracy Version 1.0. The Cochrane Collaboration, 2010. Available from srdta.cochrane.org/.

\section{McPherson 2015}

McPherson S, Hardy T, Henderson E, Burt AD, Day CP, Anstee QM. Evidence of NAFLD progression from steatosis to fibrosing-steatohepatitis using paired biopsies: implications for prognosis and clinical management. Journal of Hepatology 2015;62(5):1148-55. [PMID: 25477264]

\section{Moher 2011}

Moher D, Altman DG, Liberati A, Tetzlaff J. PRISMA statement. Epidemiology (Cambridge, Mass.) 2011;22(1):128; author reply 128. [PMID: 21150360]

\section{NICE 2016}

National Guideline Centre (UK). Non-alcoholic fatty liver disease: assessment and management. www.nice.org.uk/ guidance/ng49 (accessed prior to 2 July 2020). [PMID: 27441333]

\section{Pais 2013}

Pais R, Charlotte F, Fedchuk L, Bedossa P, Lebray P, Poynard T, et al. A systematic review of follow-up biopsies reveals disease progression in patients with non-alcoholic fatty liver. Journal of Hepatology 2013;59(3):550-6. [PMID: 23665288]

\section{Perumpail 2017}

Perumpail BJ, Khan MA, Yoo ER, Cholankeril G, Kim D, Ahmed A. Clinical epidemiology and disease burden of nonalcoholic fatty liver disease. World Journal of Gastroenterology 2017;23(47):8263-76. [PMID: 29307986]

\section{Pu 2019}

Pu K, Wang Y, Bai S, Wei H, Zhou Y, Fan J, et al. Diagnostic accuracy of controlled attenuation parameter (CAP) as a noninvasive test for steatosis in suspected non-alcoholic fatty liver disease: a systematic review and meta-analysis. BMC Gastroenterology 2019;19(1):51. [PMID: 30961539]

\section{QUADAS-2}

University of Bristol. QUADAS-2. www.bristol.ac.uk/populationhealth-sciences/projects/quadas/quadas-2/ (accessed 29 June 2020).

\section{Review Manager 2014 [Computer program]}

Nordic Cochrane Centre, The Cochrane Collaboration Review Manager (RevMan). Version 5.3. Copenhagen: Nordic Cochrane Centre, The Cochrane Collaboration, 2014.

\section{Rinella 2016}

Rinella ME, Sanyal AJ. Management of NAFLD: a stage-based approach. Nature Reviews. Gastroenterology \& Hepatology 2016;13(4):196-205. [PMID: 26907882]

\section{Royle 2003}

Royle P, Milne R. Literature searching for randomized controlled trials used in Cochrane reviews: rapid versus exhaustive searches. International Journal of Technology Assessment in Health Care 2003;19(4):591-603.

\section{Sandrin 2002}

Sandrin L, Tanter M, Gennisson JL, Catheline S, Fink M. Shear elasticity probe for soft tissues with 1-D transient elastography. IEEE Transactions on Ultrasonics, Ferroelectrics, and Frequency Control 2002;49(4):436-46. [PMID: 11989699]

\section{Sasso 2010}

Sasso M, Beaugrand M, de Ledinghen V, Douvin C, Marcellin P, Poupon R, et al. Controlled attenuation parameter (CAP): a novel VCTE guided ultrasonic attenuation measurement for the evaluation of hepatic steatosis: preliminary study and validation in a cohort of patients with chronic liver disease from various causes. Ultrasound in Medicine \& Biology 2010;36(11):1825-35. [PMID: 20870345]

\section{Schuetz 2012}

Schuetz GM, Schlattmann P, Dewey M. Use of 3×2 tables with an intention to diagnose approach to assess clinical performance of diagnostic tests: meta-analytical evaluation of coronary CT angiography studies. BMJ (Clinical Research Ed.) 2012;345:e6717. [PMID: 23097549] 


\section{Schünemann 2020}

Schünemann HJ, Mustafa RA, Brozek J, Steingart KR, Leeflang M, Murad MH, et al. GRADE guidelines: 21 part 2. Test accuracy: inconsistency, imprecision, publication bias, and other domains for rating the certainty of evidence and presenting it in evidence profiles and summary of findings tables. Journal of Clinical Epidemiology 2020;122:142-52. [PMID: 32058069]

\section{Shah 2015}

Shah RV, Allison MA, Lima JA, Bluemke DA, Abbasi SA, Ouyang P, et al. Liver fat, statin use, and incident diabetes: the Multi-Ethnic Study of Atherosclerosis. Atherosclerosis 2015;242(1):211-7. [PMID: 26209814]

\section{Shi 2014}

Shi KQ, Tang JZ, Zhu XL, Ying L, Li DW, Gao J, et al. Controlled attenuation parameter for the detection of steatosis severity in chronic liver disease: a meta-analysis of diagnostic accuracy. Journal of Gastroenterology and Hepatology 2014;29(6):1149-58. [PMID: 24476011]

\section{Singh 2015}

Singh S, Allen AM, Wang Z, Prokop LJ, Murad MH, Loomba R. Fibrosis progression in nonalcoholic fatty liver vs nonalcoholic steatohepatitis: a systematic review and meta-analysis of paired-biopsy studies. Clinical Gastroenterology and Hepatology 2015;13(4):643-54.e1-9. [PMID: 24768810]

\section{Soderberg 2010}

Soderberg C, Stal P, Askling J, Glaumann H, Lindberg G, Marmur J, et al. Decreased survival of subjects with elevated liver function tests during a 28-year follow-up. Hepatology (Baltimore, Md.) 2010;51(2):595-602. [PMID: 20014114]

\section{Targher 2016}

Targher G, Marchesini G, Byrne CD. Risk of type 2 diabetes in patients with non-alcoholic fatty liver disease: causal association or epiphenomenon? Diabetes \& Metabolism 2016;42(3):142-56. [PMID: 27142870]

\section{Tiniakos 2018}

Dina G Tiniakos, Quentin M Anstee, Alastair D Burt. Fatty Liver Disease. In: Alastair D Burt, Linda D Ferrell, Stefan G Hübscher, , editors(s). Macsween's Pathology of the Liver. Seventh edition. Vol. 5. Elsevier, 2018:308-371. [DOI: 10.1016/ B978-0-7020-6697-9.00005-4.]

\section{Tognarelli 2015}

Tognarelli JM, Dawood M, Shariff MI, Grover VP, Crossey MM, Cox IJ, et al. Magnetic resonance spectroscopy: principles and techniques: lessons for clinicians. Journal of Clinical and Experimental Hepatology 2015;5(4):320-8. [PMID: 26900274]

\section{Wang 2015}

Wang Y, Fan Q, Wang T, Wen J, Wang H, Zhang T. Controlled attenuation parameter for assessment of hepatic steatosis grades: a diagnostic meta-analysis. International Journal of Clinical and Experimental Medicine 2015;8(10):17654-63. [PMID: 26770355]

\section{Wang 2018}

Wang XM, Zhang XJ, Ma L. Diagnostic performance of magnetic resonance technology in detecting steatosis or fibrosis in patients with nonalcoholic fatty liver disease: a meta-analysis. Medicine 2018;97(21):e10605. [PMID: 29794735]

\section{Whiting 2011}

Whiting PF, Rutjes AW, Westwood ME, Mallett S, Deeks JJ, Reitsma JB, et al. QUADAS-2: a revised tool for the quality assessment of diagnostic accuracy studies. Annals of Internal Medicine 2011;155(8):529-36.

\section{Wong 2018}

Wong VW, Chan WK, Chitturi S, Chawla Y, Dan YY, Duseja A, et al. Asia-Pacific working party on non-alcoholic fatty liver disease guidelines 2017 - part 1: definition, risk factors and assessment. Journal of Gastroenterology and Hepatology 2018;33(1):70-85. [PMID: 28670712]

\section{Younossi 2016}

Younossi ZM, Koenig AB, Abdelatif D, Fazel Y, Henry L, Wymer M. Global epidemiology of nonalcoholic fatty liver disease-Metaanalytic assessment of prevalence, incidence, and outcomes. Hepatology (Baltimore, Md.) 2016;64(1):73-84. [PMID: 26707365]

\section{ADDITIONAL TABLES}

Table 1. Conversion grid: histopathological scoring systems for NAFLD

\begin{tabular}{lllll}
\hline Feature & $\begin{array}{l}\text { Grade } \\
\text { range, ac- } \\
\text { cording } \\
\text { to SAF* }\end{array}$ & Brunt 1999 & $\begin{array}{l}\text { NAS (Kleiner 2005) plus fi- } \\
\text { brosis }\end{array}$ & SAF* (Bedossa 2014) $^{*}$ \\
\hline $\begin{array}{l}\text { Steatosis (per- } \\
\text { centage of hepa- } \\
\begin{array}{l}\text { tocytes with fat } \\
\text { accumulation) }\end{array}\end{array}$ & S0 & $0-10 \%$ & $0-5 \%$ & $0-5 \%$ \\
\cline { 2 - 5 } & S1 & $10-33 \%$ & $5-33 \%$ & $5-33 \%$ \\
\cline { 2 - 5 } & S2 & $34-66 \%$ & $34-66 \%$ & $34-66 \%$ \\
\hline
\end{tabular}


Table 1. Conversion grid: histopathological scoring systems for NAFLD

\begin{tabular}{|c|c|c|c|c|}
\hline & S3 & $>66 \%$ & $>66 \%$ & $>66 \%$ \\
\hline \multirow{4}{*}{$\begin{array}{l}\text { Lobular inflam- } \\
\text { mation (number } \\
\text { of foci of inflam- } \\
\text { matory cells with- } \\
\text { in the lobule) }\end{array}$} & \multirow[t]{2}{*}{0} & 0 & 0 & \multirow[t]{2}{*}{0} \\
\hline & & $<2$ & $1-2$ & \\
\hline & \multirow[t]{2}{*}{1} & $2-4$ & $2-4$ & \multirow[t]{2}{*}{$>2$} \\
\hline & & $>4$ & $>4$ & \\
\hline \multirow{3}{*}{$\begin{array}{l}\text { Ballooning of he- } \\
\text { patocytes }\end{array}$} & 0 & None/minimal & None & Normal hepatocytes \\
\hline & 1 & Present-zone 3 & Few & $\begin{array}{l}\text { Clusters of hepatocytes of normal size, } \\
\text { but with a rounded shape and pale cyto- } \\
\text { plasm }\end{array}$ \\
\hline & 2 & Marked-zone 3 & Many & $\begin{array}{l}\text { Same as grade } 1 \text { with some enlarged he- } \\
\text { patocytes, at least } 2 \text {-fold that of normal } \\
\text { cells }\end{array}$ \\
\hline \multirow[t]{5}{*}{ Fibrosis } & Fo & None & None & None \\
\hline & $\mathrm{F} 1$ & $\begin{array}{l}\text { Perisinusoidal or } \\
\text { portal fibrosis }\end{array}$ & $\begin{array}{l}\text { 1: perisinusoidal or periportal } \\
\text { 1A: mild, zone } 3 \text {, perisinusoidal } \\
\text { 1B: moderate, zone } 3 \text {, perisi- } \\
\text { nusoidal } \\
\text { 1C: portal/periportal }\end{array}$ & Perisinusoidal or portal fibrosis \\
\hline & $\mathrm{F} 2$ & $\begin{array}{l}\text { Perisinusoidal and } \\
\text { portal/periportal fi- } \\
\text { brosis }\end{array}$ & $\begin{array}{l}\text { Perisinusoidal and portal/peri- } \\
\text { portal fibrosis }\end{array}$ & $\begin{array}{l}\text { Perisinusoidal and periportal fibrosis } \\
\text { without bridging }\end{array}$ \\
\hline & F3 & Bridging fibrosis & Bridging fibrosis & Bridging fibrosis \\
\hline & F4 & Cirrhosis & Cirrhosis & Cirrhosis \\
\hline
\end{tabular}

NAS: NAFLD activity score; SAF: steatosis, activity, fibrosis.

Table 2. Controlled attenuation parameter staging and proposed values

\begin{tabular}{lllll}
\hline Steatosis stage & $\begin{array}{l}\text { Optimal cut-off } \\
\text { Sasso } 2010\end{array}$ & $\begin{array}{l}\text { Optimal cut-off } \\
\text { Shi } 2014\end{array}$ & $\begin{array}{l}\text { Median cut-off } \\
\text { Wang } 2015\end{array}$ & $\begin{array}{l}\text { Optimal cut-off } \\
\text { Karlas 2017 }\end{array}$ \\
\hline S1 (mild steatosis) & $237.7 \mathrm{~dB} / \mathrm{m}$ & $232.5 \mathrm{~dB} / \mathrm{m}$ & $238 \mathrm{~dB} / \mathrm{m} \mathrm{and} 250 \mathrm{~dB} / \mathrm{m}$ & $248 \mathrm{~dB} / \mathrm{m}$ \\
\hline $\mathrm{SE}$ & 0.91 & 0.78 & 0.78 & 0.688 \\
\hline $\mathrm{SP}$ & 0.81 & 0.79 & 0.79 & 0.822 \\
\hline $\mathrm{S} 2$ (moderate steatosis) & $259.4 \mathrm{~dB} / \mathrm{m}$ & $255 \mathrm{~dB} / \mathrm{m}$ & $259 \mathrm{~dB} / \mathrm{m}$ and $263 \mathrm{~dB} / \mathrm{m}$ & $268 \mathrm{~dB} / \mathrm{m}$ \\
\hline $\mathrm{SE}$ & 0.89 & 0.85 & 0.82 & 0.773 \\
\hline $\mathrm{SP}$ & 0.86 & 0.79 & 0.79 & 0.812 \\
\hline
\end{tabular}

Transient elastography with controlled attenuation parameter (CAP) for diagnosis of moderate or severe steatosis in people with 
Table 2. Controlled attenuation parameter staging and proposed values (Continued)

\begin{tabular}{lllll} 
S3 (severe steatosis) & $292.3 \mathrm{~dB} / \mathrm{m}$ & $290 \mathrm{~dB} / \mathrm{m}$ & $290 \mathrm{~dB} / \mathrm{m}$ & $280 \mathrm{~dB} / \mathrm{m}$ \\
\hline $\mathrm{SE}$ & 1 & 0.83 & 0.86 & 0.882 \\
\hline $\mathrm{SP}$ & 0.78 & 0.79 & 0.89 & 0.776 \\
\hline
\end{tabular}

S1: mild steatosis (5\% or $10 \%$ to $33 \%$ (depending on scale used)); S2: moderate steatosis (34\% to $66 \%$ ); S3: severe steatosis (greater than $66 \%$ ) (Index test(s)) (Table 1); SE: sensitivity; SP: specificity; dB/m: decibel/milliwatt.

Table 3. Comparative analysis of recommendations for the diagnosis of non-alcoholic fatty liver disease

\begin{tabular}{|c|c|c|c|c|c|}
\hline & EASL & NICE & Asia-Pacific & AISF & AASLD \\
\hline $\begin{array}{l}\text { Systematic diagnosis of } \\
\text { NAFLD }\end{array}$ & No & No & No & No & No \\
\hline $\begin{array}{l}\text { Diagnosis of NAFLD in high- } \\
\text { risk groups }\end{array}$ & $\begin{array}{l}\text { Yes } \\
\text { Metabolic risk factors } \\
\text { Abnormal liver enzymes }\end{array}$ & $\begin{array}{l}\text { Yes } \\
\text { Type } 2 \\
\text { diabetes }\end{array}$ & $\begin{array}{l}\text { Yes } \\
\text { Type } 2 \text { dia- } \\
\text { betes }\end{array}$ & $\begin{array}{l}\text { Not } \\
\text { men- } \\
\text { tioned }\end{array}$ & $\begin{array}{l}\text { 'Active surveillance' suggested } \\
\text { for people with type } 2 \text { diabetes } \\
\text { mellitus. }\end{array}$ \\
\hline
\end{tabular}

AASLD: American Association for the Study of Liver Diseases (Chalasani 2018); Asia-Pacific: Asia-Pacific Working Party (Chitturi 2018; Wong 2018); AISF: Italian Association for the Study of the Liver (AISF 2017); EASL: European Association for the Study of the Liver (EASL Guidelines 2016); NICE: National Institute for Health and Care Excellence (NICE 2016).

Table 4. Semi-quantitative histopathological scoring systems for progression of fibrosis to cirrhosis. Conversion grid for the stages of hepatic fibrosis

\section{Stage of estimated fibrosis following:}

\begin{tabular}{lll}
\hline METAVIR (French METAVIR CSG 1994) & Ishak 1995 & Kleiner 2005 \\
\hline F0 & F0 & F0 \\
\hline F1 & F1 & F1 \\
\hline F1 & F2 & F1 \\
\hline F2 & F3 & F2 \\
\hline F3 & F4 & F3 \\
\hline F4 & F5 & F4 \\
\hline F4 & F6 & \\
\hline
\end{tabular}

F: stage of hepatic fibrosis; F0: no fibrosis; F1: portal fibrous expansion; F2: thin fibrous septa emanating from portal triads; F3: fibrous septa bridging portal triads and central veins; F4: cirrhosis. Clinically significant fibrosis is generally defined as F2 or greater (on the METAVIR scale from F0 to F4 with F4 being cirrhosis). 
Table 5. Data extraction form

\begin{tabular}{|c|c|}
\hline Domain & Items \\
\hline \multirow[t]{7}{*}{ Study characteristics } & Author \\
\hline & Journal \\
\hline & Publication year \\
\hline & Language \\
\hline & Study design \\
\hline & Start and finish dates of study \\
\hline & Study location (country) \\
\hline \multirow[t]{9}{*}{ Study population } & Number of participants meeting the criteria \\
\hline & Total number of participants assessed \\
\hline & Age distribution (mean/median, standard deviation, range) \\
\hline & Sex distribution \\
\hline & Study location \\
\hline & Presence or absence of diabetes mellitus \\
\hline & Serum levels of alanine transferase \\
\hline & Steatosis stage distribution \\
\hline & BMI \\
\hline \multirow[t]{7}{*}{ Index test } & Type of index test's probe used (M or XL) \\
\hline & Cut-off level used for staging of steatosis, $\mathrm{dB} / \mathrm{m}$ \\
\hline & Blinding of operators for results of histopathological analysis \\
\hline & The validity of the measurements (Number of valid measurements \\
\hline & Success rate, IQR/med) \\
\hline & Levels of liver stiffness, $\mathrm{kPa}$ \\
\hline & Missing results for index test \\
\hline \multirow[t]{4}{*}{ Reference test } & Blinding of pathologists to results of TE with CAP \\
\hline & Severity of steatosis, $\%$ \\
\hline & Missing results for reference standard \\
\hline & Levels of histological activity (from 0 to 4 ) \\
\hline
\end{tabular}


Table 5. Data extraction form (Continued)

\begin{tabular}{ll} 
Flow and timing & Time interval between index test(s) and reference test (weeks) \\
\hline Study results & True positives for each presented cut-off value \\
\cline { 2 - 2 } & False positives for each presented cut-off value \\
\cline { 2 - 2 } & True negatives for each presented cut-off value \\
\cline { 2 - 3 } & False negatives for each presented cut-off value \\
\hline Correspondence with authors & Source of funding \\
\hline Funding & $\begin{array}{l}\text { Are study authors affiliated with the manufacturer of the index test? } \\
\text { Is the study directly funded by the manufacturer? } \\
\text { Have study authors reported conflicts of interests related to the manufacturer or other funding } \\
\text { sources? }\end{array}$
\end{tabular}

BMI: body mass index; $\mathrm{dB} / \mathrm{m}$ : decibel/milliwatt; IQR/med: interquartile range/median: $\mathrm{kPa}$ : kilopascal; TE with CAP: transient elastography with controlled attenuation parameter.

\section{A P PE N D I CES}

Appendix 1. Search strategy

Transient elastography with controlled attenuation parameter (CAP) for diagnosis of moderate or severe steatosis in people with non-alcoholic fatty liver disease

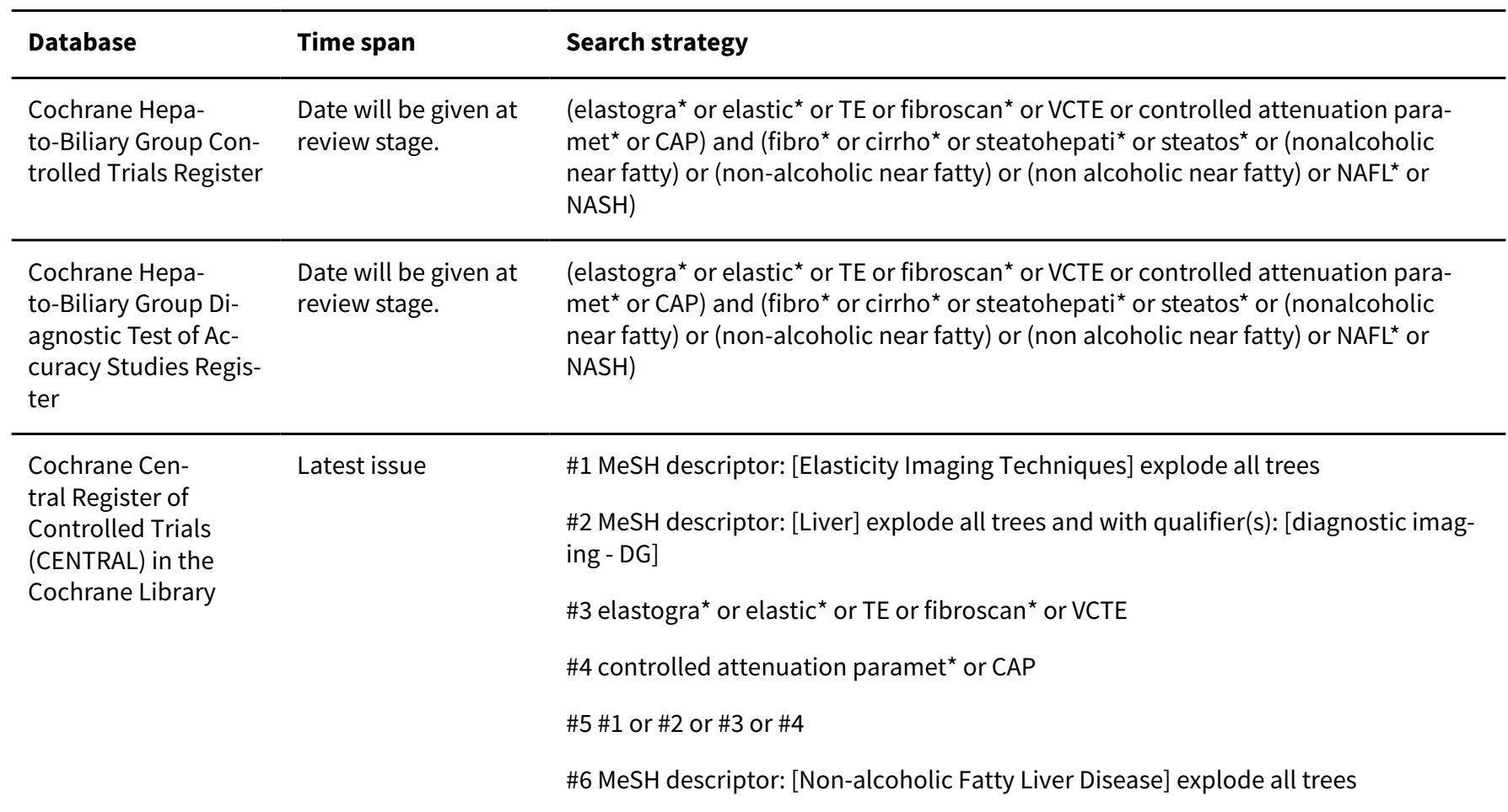

Transient elastography with controlled attenuation parameter (CAP) for diagnosis of moderate or severe steatosis in people with 


\author{
\#7 MeSH descriptor: [Fatty Liver] explode all trees \\ \#8 MeSH descriptor: [Liver Cirrhosis] explode all trees \\ \#9 MeSH descriptor: [Fibrosis] explode all trees \\ \#10 (fibro* or cirrho* or steatohepati* or steatos* or non?alcoholic near fatty or \\ $\mathrm{NAFL}^{*}$ or NASH) \\ $\# 11$ \#6 or \#7 or \#8 or \#9 or \#10 \\ $\# 12 \# 5$ and \#11
}

$\begin{array}{ll}\text { MEDLINE Ovid } & \begin{array}{l}1946 \text { to the date of } \\ \text { the search }\end{array} \\ & \begin{array}{l}\text { 1. exp Elasticity Imaging Techniques/ } \\ \text { 2. exp Liver/dg [Diagnostic Imaging] }\end{array} \\ & \begin{array}{l}\text { 3. (elastogra* or elastic }{ }^{*} \text { or TE or fibroscan* or VCTE).mp. [mp=title, abstract, origi- } \\ \text { nal title, name of substance word, subject heading word, floating sub-heading word, } \\ \text { keyword heading word, organism supplementary concept word, protocol supple- } \\ \text { mentary concept word, rare disease supplementary concept word, unique identifi- } \\ \text { er, synonyms] }\end{array}\end{array}$

4. (controlled attenuation paramet* or CAP).mp. [mp=title, abstract, original title, name of substance word, subject heading word, floating sub-heading word, keyword heading word, organism supplementary concept word, protocol supplementary concept word, rare disease supplementary concept word, unique identifier, synonyms]

5. 1 or 2 or 3 or 4

6. exp Non-alcoholic Fatty Liver Disease/

7. exp Fatty Liver/

8. exp Liver Cirrhosis/

9. exp Fibrosis/

10. (fibro* or cirrho* or steatohepati* or steatos* or (non?alcoholic adj fatty) or $\mathrm{NAFL}^{*}$ or NASH).mp. [mp=title, abstract, original title, name of substance word, subject heading word, floating sub-heading word, keyword heading word, organism supplementary concept word, protocol supplementary concept word, rare disease supplementary concept word, unique identifier, synonyms]

\title{
11.6 or 7 or 8 or 9 or 10
}

\section{5 and 11}

13. limit 12 to ("adolescent (13 to 18 years)" or "young adult (19 to 24 years)" or "adult (19 to 44 years)" or "young adult and adult (19-24 and 19-44)" or "middle age (45 to 64 years)" or "middle aged (45 plus years)" or "all aged (65 and over)" or "aged (80 and over)")

\begin{tabular}{ll}
\hline $\begin{array}{l}\text { Embase Ovid } \\
\text { the search }\end{array}$ & $\begin{array}{l}\text { 1. exp transient elastography/ } \\
\text { 2. exp elastograph/ }\end{array}$ \\
& $\begin{array}{l}\text { 3. (elastogra* or elastic* or TE or fibroscan* or VCTE).mp. [mp=title, abstract, head- } \\
\text { ing word, drug trade name, original title, device manufacturer, drug manufacturer, } \\
\text { device trade name, keyword, floating subheading word, candidate term word] }\end{array}$ \\
& $\begin{array}{l}\text { 4. (controlled attenuation paramet* or CAP).mp. [mp=title, abstract, heading word, } \\
\text { drug trade name, original title, device manufacturer, drug manufacturer, device } \\
\text { trade name, keyword, floating subheading word, candidate term word] }\end{array}$
\end{tabular}

Transient elastography with controlled attenuation parameter (CAP) for diagnosis of moderate or severe steatosis in people with 


\begin{abstract}
5. 1 or 2 or 3 or 4
6. exp nonalcoholic fatty liver/

7. exp fatty liver/

8. exp liver cirrhosis/

9. exp liver fibrosis/

10. (fibro* or cirrho* or steatohepati* or steatos* or (non?alcoholic adj fatty) or $\mathrm{NAFL}^{*}$ or NASH).mp. [mp=title, abstract, heading word, drug trade name, original title, device manufacturer, drug manufacturer, device trade name, keyword, floating subheading word, candidate term word]
\end{abstract}

11.6 or 7 or 8 or 9 or 10

12. 5 and 11

13. limit 12 to (adult $<18$ to 64 years $>$ or aged $<65+$ years $>$ )

\begin{tabular}{|c|c|c|}
\hline LILACS (Bireme) & $\begin{array}{l}1982 \text { to the date of } \\
\text { the search }\end{array}$ & $\begin{array}{l}\text { elastogra\$ or elastic\$ or TE or fibroscan\$ or VCTE [Words] and (fibro\$ or cirrho\$ or } \\
\text { steatohepati\$ or steatos\$ or non\$alcoholic fatty or NAFL\$ or NASH) [Words] }\end{array}$ \\
\hline \multirow[t]{2}{*}{$\begin{array}{l}\text { Science Citation In- } \\
\text { dex Expanded (Web } \\
\text { of Science) }\end{array}$} & $\begin{array}{l}1900 \text { to the date of } \\
\text { the search }\end{array}$ & $\begin{array}{l}\text { \#3 \#2 AND \#1 } \\
\text { \#2 TI=(fibro* or cirrho* or steatohepati* or steatos* or (non?alcoholic near fatty) or } \\
\mathrm{NAFL}^{\star} \text { or NASH) }\end{array}$ \\
\hline & & $\begin{array}{l}\# 1 \mathrm{Tl}=\left(\text { elastogra* or elastic }{ }^{\star} \text { or TE or fibroscan* or VCTE or (controlled attenuation }\right. \\
\text { paramet }^{\star} \text { ) or CAP) }\end{array}$ \\
\hline \multirow[t]{2}{*}{$\begin{array}{l}\text { Conference Proceed- } \\
\text { ings Citation Index } \\
\text { - Science (Web of } \\
\text { Science) }\end{array}$} & $\begin{array}{l}1990 \text { to the date of } \\
\text { the search }\end{array}$ & $\begin{array}{l}\text { \#3 \#2 AND \#1 } \\
\text { \#2 TI=(fibro* or cirrho* or steatohepati* or steatos* or (non?alcoholic near fatty) or } \\
\mathrm{NAFL}^{\star} \text { or NASH) }\end{array}$ \\
\hline & & $\begin{array}{l}\# 1 \mathrm{TI}=\left(\text { elastogra* }^{\star} \text { or elastic* or TE or fibroscan* or VCTE or (controlled attenuation }\right. \\
\left.\text { paramet }^{\star}\right) \text { or CAP) }\end{array}$ \\
\hline
\end{tabular}

Appendix 2. QUADAS-2

\begin{tabular}{|c|c|c|c|c|}
\hline Domain & 1. Participant selection & 2. Index test & 3. Reference standard & 4. Flow and timing \\
\hline $\begin{array}{l}\text { Sig- } \\
\text { nalling } \\
\text { questions } \\
\text { and crite- } \\
\text { ria }\end{array}$ & $\begin{array}{l}\text { Q1: "Was a consecutive or } \\
\text { random sample of partici- } \\
\text { pants enrolled?" } \\
\text { Yes - If the study reports } \\
\text { on a consecutive or a ran- } \\
\text { dom selection of partici- } \\
\text { pants. } \\
\text { No - if the study reports } \\
\text { on another form of selec- } \\
\text { tion of participants. }\end{array}$ & $\begin{array}{l}\text { Q1: "Were the index test re- } \\
\text { sults interpreted without } \\
\text { knowledge of the results of } \\
\text { the reference standard?" } \\
\text { Yes - if the study reports } \\
\text { that the results of the index } \\
\text { test were interpreted with- } \\
\text { out the knowledge of the re- } \\
\text { sults of the reference stan- } \\
\text { dard. } \\
\text { No - if the study reports } \\
\text { that results of the index } \\
\text { test were interpreted with }\end{array}$ & $\begin{array}{l}\text { Q1: "Is the reference stan- } \\
\text { dard likely to correctly clas- } \\
\text { sify the target condition?" } \\
\text { Yes - this item will always } \\
\text { be 'Yes' because a correct } \\
\text { reference standard is part of } \\
\text { the inclusion criteria of this } \\
\text { review. } \\
\text { No - not applicable. } \\
\text { Unclear - not applicable. }\end{array}$ & $\begin{array}{l}\text { Q1: "Was there an appro- } \\
\text { priate interval between the } \\
\text { index test and the refer- } \\
\text { ence standard?" } \\
\text { Yes - if the interval be- } \\
\text { tween the index test and } \\
\text { the reference standard } \\
\text { was }<3 \text { months. } \\
\text { No - if the interval was }>3 \\
\text { months. } \\
\text { Unclear - if the study does } \\
\text { not report the interval be- }\end{array}$ \\
\hline
\end{tabular}

Transient elastography with controlled attenuation parameter (CAP) for diagnosis of moderate or severe steatosis in people with 
(Continued)

Unclear - if the study does not report on how the participants were enrolled.

Q2: "Did the study avoid inappropriate exclusions?"

Yes - if definitions of exclusion criteria are inappropriate (e.g. difficult to diagnose participants, failure at liver biopsy, failure on transient elastography with controlled attenuation parameter) and all exclusions are reported.

No - if exclusion criteria are inappropriate and exclusions are not reported.

Unclear - if the study does not report causes of exclusions. the results of the reference standard.

Unclear - if the study does not report information about blinding of the results of the index test and reference standard.

Q2: "If a threshold was used, was it prespecified?"

Yes - the cut-off of transient elastography with controlled attenuation parameter for specific steatosis stage was prespecified.

No - it was not.

Unclear - there was no such information.
Q2: "Were the results of liver biopsy interpreted without knowledge of the results of transient elastography with controlled attenuation parameter?"

Yes - if the study reports that the results of the reference standard were interpreted without the knowledge of the results of the index test.

No - if the study reports that the results of the reference standard were interpreted with the knowledge of the results of the index test.

Unclear - if the study does not report information about blinding of the results of the reference standard and the index test. tween the index test and the reference standard.

For studies with bariatric interventions:

Yes - if the interval between the index test and the reference standard was $\leq 1$ month.

No - if the interval was $>1$ month.

Unclear - if the study does not report the interval between the index test and the reference standard.

Q2: "Did all participants receive the same reference standard (liver biopsy)?"

Yes - if the study has only 1 reference standard for all the participants

No - if the study has $>1$ reference standard.

Unclear - if the study information regarding the use of reference standard are unclear

Q3: "Were all participants included in the analysis and analysed according to intention-to-diagnose principle (non-evaluable results considered as false)?"

Yes - if all enrolled participants were included in the analysis and non-evaluable index test results were analysed according to the intention-to-diagnose principle.

No - if any participant was excluded from the analysis for any reason or nonevaluable index test results were not analysed according to intention-todiagnose principle.

Unclear - if the exclusion of participants from the analysis is unclear. 
(Continued)

Risk of bias
Could the selection of participants have introduced bias?

Low risk: 'Yes' for all signalling questions.

High risk: 'No' or 'Unclear' for $\geq 1$ signalling question.
Could the conduct or interpretation of the index test have introduced bias?

Low risk: 'Yes' for all signalling questions.

High risk: 'No' or 'Unclear' for $\geq 1$ signalling question.
Could the reference standard, its conduct, or its interpretation have introduced bias?

Low risk: 'Yes' for all signalling questions.

High risk: 'No' or 'Unclear' for $\geq 1$ signalling question.
Could the participant flow have introduced bias?

Low risk: 'Yes' for all signalling questions.

High risk: 'No' or 'Unclear' for $\geq 1$ signalling question.

\section{Concerns Are there concerns that in- about ap- cluded participants and plicabili- setting do not match the ty \\ review question?}

Low concern: there was low concern that the included participants did not match the review question.

High concern: there was high concern that the included participants did not match the review question.

Unclear concern: if it was unclear.
Are there concerns that the index test, its conduct, or interpretation differ from the review question?

Low concern: the index test, its conduct, or its interpretation does not differ from the way it is used in clinical practice.

High concern: the index test, its conduct, or its interpretation differs from the way it is used in clinical practice.

Unclear concern: if it was unclear.
Are there concerns that the target condition as defined by the reference standard does not match the question?

High concern: all participants did not undergo liver biopsy for steatosis.

Low concern: all participants underwent liver biopsy for steatosis.

Unclear concern: if it was unclear.

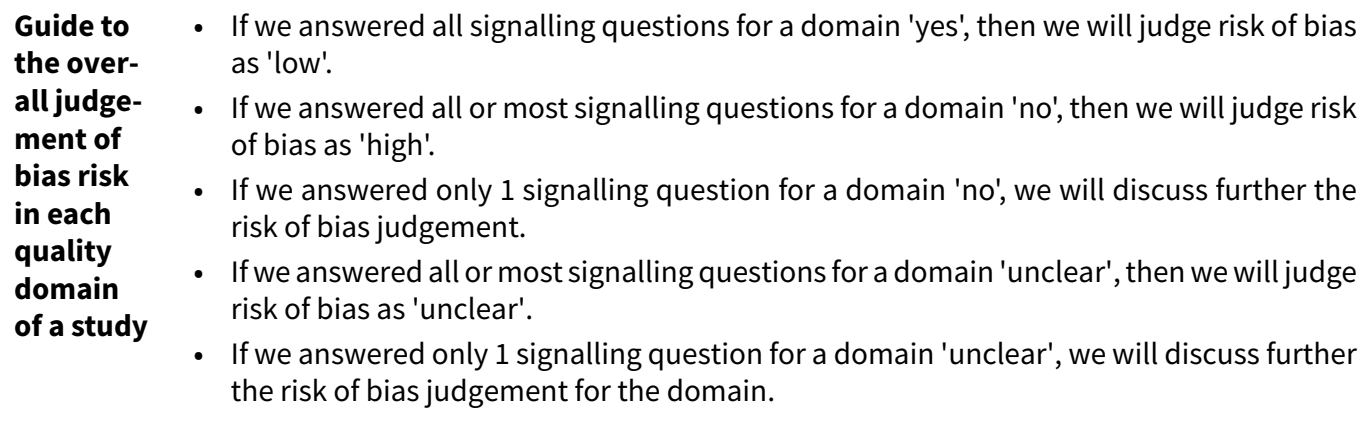

- If we answered all or most signalling questions for a domain 'unclear', then we will judge risk of bias as 'unclear'.

- If we answered only 1 signalling question for a domain 'unclear', we will discuss further the risk of bias judgement for the domain.

WHAT' S NEW

\begin{tabular}{lll}
\hline Date & Event & Description \\
\hline 7 August 2020 & Amended & $\begin{array}{l}\text { Missed acknowledgement added: the Russian Foundation for Ba- } \\
\text { sic Research (RFBR), Russia }\end{array}$ \\
\hline
\end{tabular}

\section{HISTORY}

Protocol first published: Issue 7, 2020 


\section{CONTRIBUTIONS OF AUTHORS}

Conceiving the protocol: TT, $C P, K S G, A B, O B$. Designing the protocol: $T T, C P, K S G, A B, O B$.

Co-ordinating the protocol: $\mathrm{CP}, \mathrm{KSG}, \mathrm{AS}$.

Designing search strategies: TT, CP, KSG.

Writing the protocol: TT, OB.

All authors approved of the final version.

\section{DECLARATIONS OF INTEREST}

TT: none.

$\mathrm{OB}$ : none.

$A B$ : none.

AS: none.

KUG: none.

CSP: none.

\section{SOURCES OF SUPPORT}

\section{Internal sources}

- I.M. Sechenov First Moscow State Medical University (Sechenov University), Russian Federation a salary

\section{External sources}

- No sources of support supplied 